\title{
Refractory Malignant Female Reproductive System Neoplasm
}

National Cancer Institute

\section{Source}

National Cancer Institute. Refractory Malignant Female Reproductive System Neoplasm.

NCI Thesaurus. Code C150527.

Malignant female reproductive system neoplasm that is resistant to treatment. 